\title{
An Information System Supporting WFD Reporting
}

\author{
Thorsten Hell ${ }^{1}$, Eckhard Kohlhas ${ }^{2}$, Sascha Schlobinski ${ }^{1}$, \\ Ralf Denzer ${ }^{3}$, and Reiner Güttler ${ }^{3}$ \\ ${ }^{1}$ Cismet GmbH, Altenkesseler Strasse 17, 66117 Saarbrücken, Germany \\ \{thorsten.hell, sascha.schlobinski\}@cismet.de \\ ${ }^{2}$ Landesamt für Umwelt, Naturschutz und Geologie Mecklenburg-Vorpommern (LUNG), \\ Goldberger Straße 1218273 Güstrow, Germany \\ eckhard.kohlhas@lung.mv-regierung.de \\ ${ }^{3}$ Environmental Informatics Group (EIG), Goebenstr. 40, 66117 Saarbrücken, Germany \\ ralf.denzer@enviromatics.org, reiner.guettler@enviromatics.net
}

\begin{abstract}
The EU Water Framework Directive (WFD) defines the quality goals for European water bodies until 2027. Water bodies are managed on very different governmental levels in the member states. Due to the federal system, Germany has a complex structure of responsibilities regarding the management and maintenance of water bodies in Europe.

The paper will present a highly integrated information system to support water body management in the context of the WFD. The system was designed and developed for the German federal state Mecklenburg-Vorpommern. The objective was to provide a state-wide information platform accessible through an environmental information system (EIS) for all activities related to the water body management, including the maintenance of the water body network itself, managing related information regarding statistics, agriculture and environmental protection, as well as water body quality like chemical and biological status.

This EIS integrates disparate data sources from a large number of individual providers through standardized services. Moreover, the EIS provides a homogenous view on very different data regarding their topic, content, structure and spatial reference and supports all WFD reporting related workflows.
\end{abstract}

Keywords: Water Framework Directive, WFD, water reporting, environmental information system, EIS.

\section{Software Support in the Context of the EU WFD}

The EU Water Framework Directive ${ }^{1}$ (WFD, [1]) requires the EU Member States to provide reports on the biological and chemical status of ground and surface water, to plan and execute programs for improvement and to report about plans, measures and results as well. In Germany these reporting requirements are managed by the federal states and the national government. The reporting is carried out through a formalized

1 http: / /ec .europa.eu/environment/water/water-framework/ 
data model in order to harmonize data across Europe. Reported information is available in the Water Information System for Europe (WISE) [5].

In the German state Mecklenburg-Vorpommern, the WFD reporting is carried out in the context of the FIS Water MV (Water Information System of MecklenburgVorpommern), which aims at providing an integrated water information base for all water-related management and reporting activities in the state. Besides water information, data from sources like general statistics, agriculture, land use, nature protection and bio diversity etc. are relevant for the assessment of water body quality.

The diverse water-related data and processes (like runoff, drainage, gages, flood forecasts, maintenance plans etc.) require the same baseline and common base geometries. For this reason, the WFD reporting cannot be regarded as an isolated activity that happens once a year.

The FIS Water MV (Fig. 1) is operated by the "Landesamt für Umwelt, Naturschutz und Geologie Mecklenburg-Vorpommern" (LUNG), the state agency for environment, nature protection and geology. The different systems are at different stages of realisation.

While the FIS WRRL (WFD Reporting) is productive, other components are under development or only at a conceptual stage. E.g. the FIS for flood risk management is currently under implementation and the information system for water use and wastewater will be upgraded from 2014 on.

All systems operate on catchments and water bodies regardless of political boundaries. They use a common water network with unified numbering and stationing, the same references for coastal zones, the same geometry and referencing for still waters and a com-

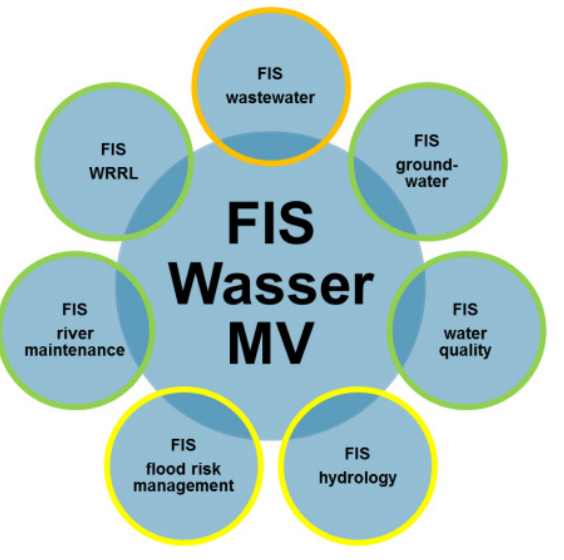

Fig. 1. Overview of FIS Water MV mon spatial reference system.

This paper gives an overview of the IS WRRL (Information System for WFD Reporting).

\section{Users, Requirements and Functional Architecture}

The FIS WRRL must support users from several organisations involved in WFD activities that have a variety of different requirements (Fig. 2). The organisatons are:

- the LUNG as the responsible organisation,

- subsidiary water administrations, namely 4 state agencies for agriculture and environment who are responsible for the management of 4 territories (shortly called "state agencies" in this paper),

- subsidiary lower water administrations, 
- water and soil management associations who are not direct subsidiaries and

- external users like contractors.

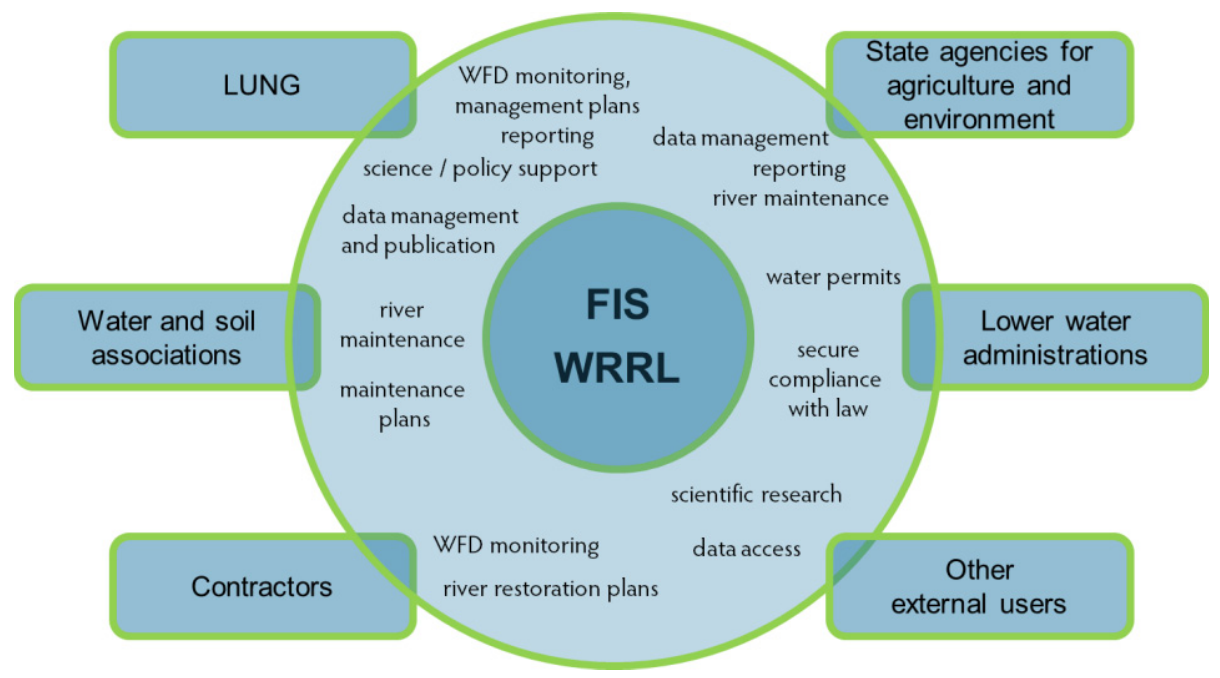

Fig. 2. Users of the FIS WRRL

Some of these users operate on the scale of the whole state, others (like lower water administrations, regions and cities) only within their territories. Also, some users work on information beyond WFD data and may need to connect their concepts and data with data in the WFD reporting system.

The following fundamental goals were defined before the FIS WRRL was developed. To:

- enable on-the-fly reporting (push-button-reporting) at any time,

- support those workflows in the LUNG which are required for WFD reporting,

- provide current and historic data on a day-to-day basis,

- hold the WFD-related data in a central common data repository in the LUNG,

- enable decentralized data input and maintenance, also by external users, while avoiding redundancies

- enable the integration of data from other data holders

- ensure that the solution is scalable,

- ensure that the solution is extendable towardsother components of the FIS Water $M V$ and beyond,

- use open standards and to avoid licensing costs.

Some of these requirements lead directly to a service-based approach, based on open source / open standards software. A critical issue was to provide a fine grain security and access control framework as users from different administrative bodies interact with the system. Fig. 3 shows an overview of the functional architecture. At the core is a WFD data repository which serves to store all WFD-related data, including photo 
documentation and related features. Beyond the data as such, information regarding management strategies, ongoing and past projects, project monitoring and cost is also included in this core repository.

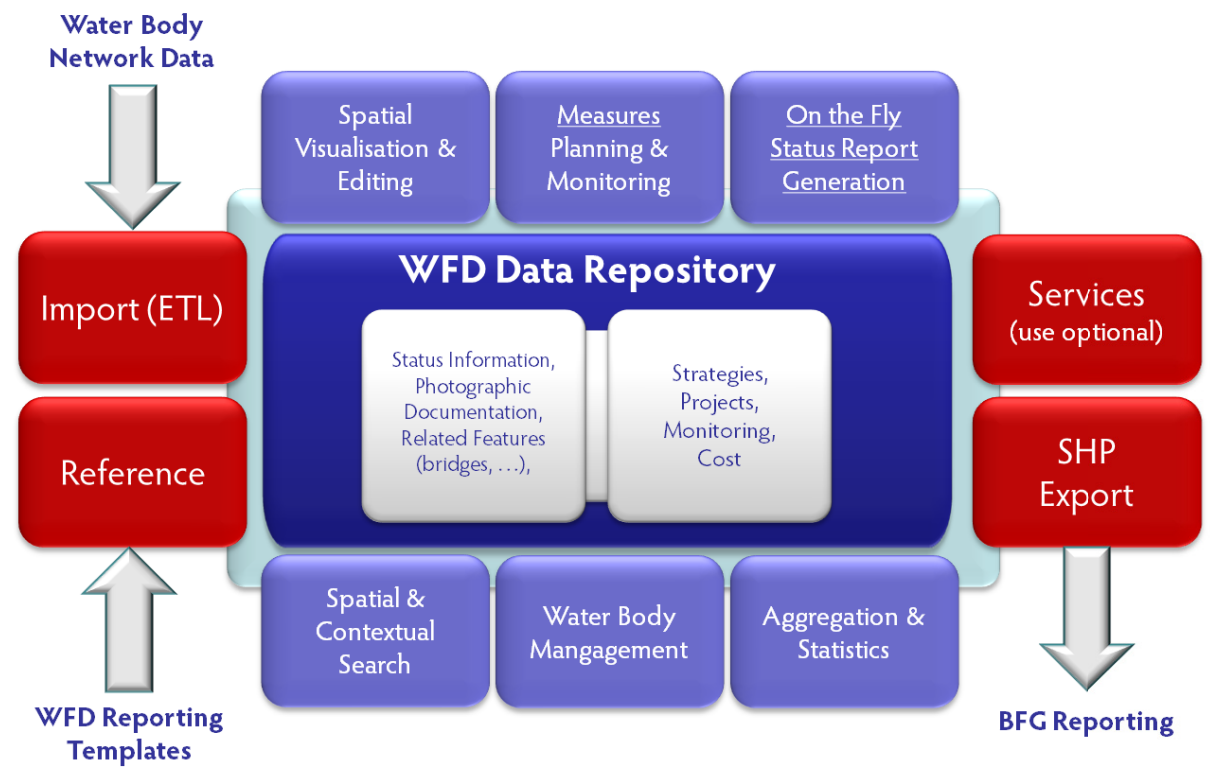

Fig. 3. Overview of functional architecture

The core repository supports users with general functionality such as:

- visualisation and editing functionality (both geography and database content),

- spatial and contextual search and

- aggregation and statistics.

Specific WFD-related functionality includes:

- water body management,

- measures planning and monitoring and

- on-thy-fly report generation.

Water body network data can be imported from external sources. The actual WFD reporting is based on WFD Reporting Templates which are used to configure the reports to the BFG (Bundesanstalt für Gewässerkunde) ${ }^{2}$. The current format for reports to the BFG is the Shape-File format ${ }^{3}$. Additionally the system allows service-based access to reports.

\footnotetext{
http: / / www.bafg.de

http://www.esri.com/library/whitepapers/pdfs/shapefile.pdf
} 


\section{Software Stack for the Solution}

The solution has been implemented with a stack of freely available open source software. The core of the solution is the CIDS product suite of CISMET GmbH. This product consists of a set of software components, application programming interfaces (APIs), management and development tools, services and applications, with a special focus on interactive solutions which need to integrate geo-spatial systems with databases, sensor networks, document-oriented systems, unstructured information sources and numerical models. CIDS is particularly suited for solutions which have to be built across existing heterogeneous information systems, which may be under control of different organisations.

CIDS uses freely available open source software exclusively, is itself freely available as open source software, distributed as Github ${ }^{4}$ projects, and is compliant with all major OGC services. CIDS has been used in numerous projects since 1999 [2]. For recent projects see $[3,4]$.

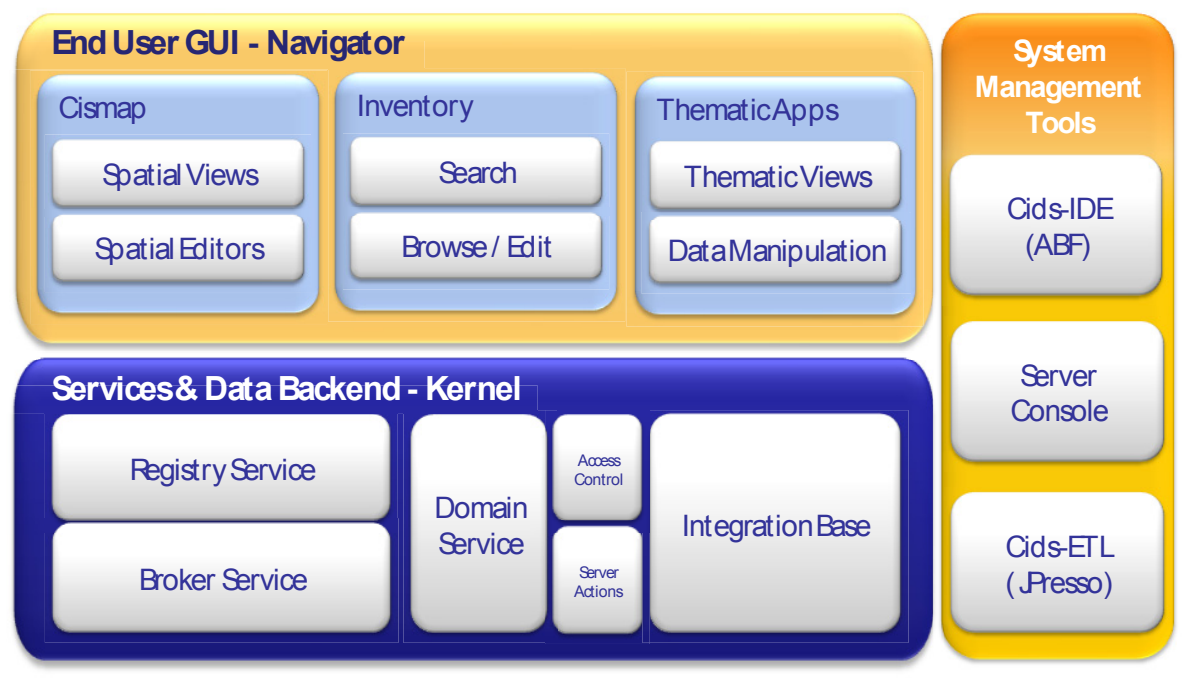

Fig. 4. Overview of CIDS product suite

The CIDS Kernel consists of one or more Integration Base(s), which are connected to a network of services. One Integration Base represents one CIDS Domain. Domains are used to organise thematic application areas within larger solutions. Domains in a large municipality can for instance be "traffic", "noise", "water bodies", "land register", "street light inventory" or "waste water". In cross-governmental applications, domains can also be organised as "City of X", "City of Y", "Water Supplier of City of Y", "Region Z". The domain concept includes fine grain control of access rights and access policies even across domains.

4 https://github.com/cismet 
The Integration Base consists of a standard relational Database Management System (DBMS) augmented by meta-information that facilitates integration tasks. It is the basis for a concrete CIDS solution and is able to model arbitrary objects (realworld objects, services, models, geographical features, other information systems, etc.), their attributes (e.g. data types, geographic location) and relationships. The Integration Base also models users, user groups, navigational structures for users, permissions, and many other properties

The Integration Base is protected through Access Control mechanisms. Moreover, the CIDS Kernel consists of a Server Actions module that can be used to run procedures on the integration base, for instance for aggregation or statistics.

The concrete data of a CIDS solution can reside in external systems, or within the Integration Base, or both. For each individual attribute, CIDS can be the "leading system" or the "following system", and there are diverse mechanisms for synchronisation and mapping of data structures in both directions (from or to an external system). As an example, in a waste water taxation application, the user interacts with a "land parcel", which in physical terms consists of geo-data coming from external WMS/WFS services, geo-data stored in the Integration Base itself (individual parcels within the premise, which are only needed in this application), ownership information from a database and documents from a standard document management system. Three types of services constitute a CIDS Service Network:

- A Domain Service publishes one Integration Base.

- A Broker Service acts as proxy hiding details of distributed access from client applications.

- A Registry Service is responsible for service name resolution, rights management, parallelised distributed search as well as core infrastructure functionalities such as service infrastructure monitoring.

A complex system can contain any number of Integration Bases and can be scaled in any direction using an appropriate number of Integration Bases and services. The physical design of this network is adapted to the organisational and computational setup of a concrete customer. A minimal system consists of one software bundle which publishes one Integration Base, and which integrates no external data sources at all (similar to operating one standard RDBMS server and using a dedicated database client software).

A CIDS Service Network is configured and developed with an interactive development and configuration environment $(\boldsymbol{C I D S}-\boldsymbol{I D E})$, which consists of the configuration and development tool $\boldsymbol{A B F}$ and the ETL tool JPresso for synchronisation with external systems. The Server Console is a management interface to the CIDS services.

As open source software the CIDS platform uses many open source tools to provide its functionality. Among the most prominent are: Netbeans $\mathrm{RCP}^{5}$ and PostGres/PostGis ${ }^{6}$.

\footnotetext{
http://netbeans.org/features/platform

http: //www.postgresql.org
} 


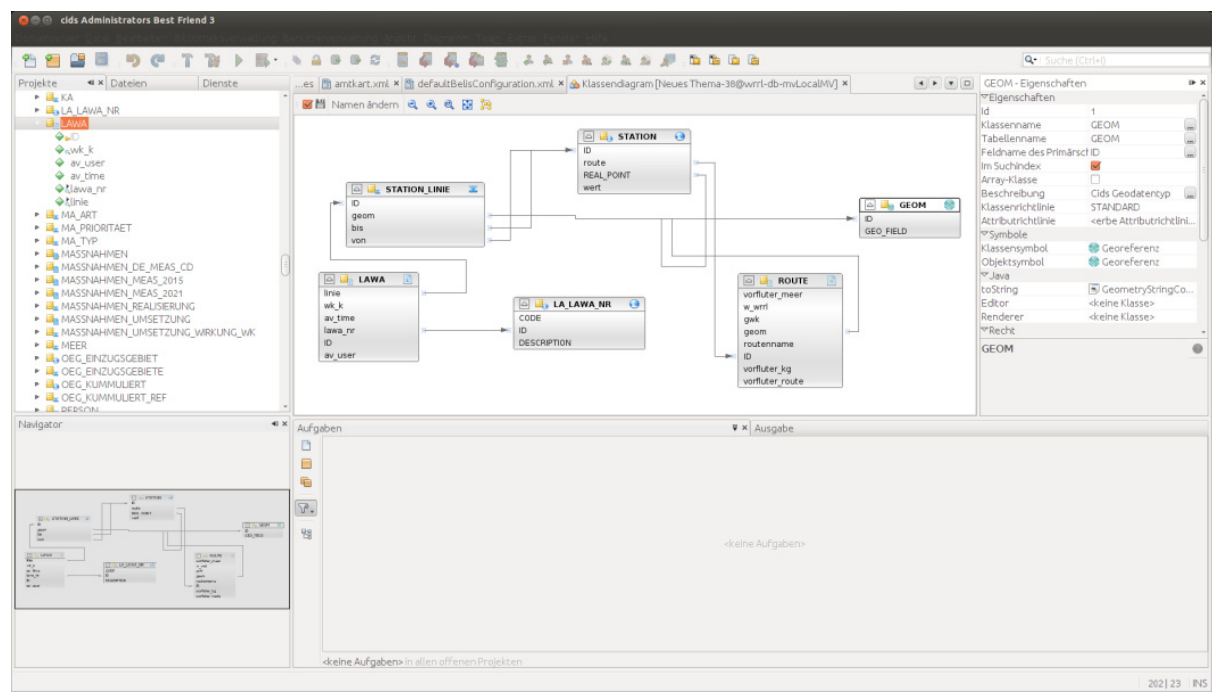

Fig. 5. CIDS ABF

\section{End User View of a Highly Integrated Interactive Application}

End users interact with the CIDS solution through customized interactive applications. At the core of most developments is the CIDS Navigator. The Navigator offers different metaphors to interact with the system. The Inventory at the left (in Fig.6) is a customized poly-hierarchical view over user interests and serves for "browsing" and "search". Entries in the inventory are often data objects that represent arbitrary data sets ("water bodies" as top level of a water domain), but can also be information like organisational processes ("maintenance", "people", "billing") or interactions with and results of numerical models ("runoff model", "model configuration", "model run"). The Inventory can be customized for user groups and individual users.

In the case of the WFD reporting system described in this paper, the inventory contains the following themes: chemical monitoring sites, biological monitoring sites, rivers, still waters, ground water bodies, coastal water bodies, buildings, projects, drainage pipes, routes, catchments, photo documentation, land use, protected areas, hydrology and several waterway maintenance themes.

For spatial information, the map component Cismap is provided. This component is a map application specialized to access OGC compliant services like WFS and WMS for information from internal and external geospatial data sets. The individual layers being visualized can be either layers generated by CIDS or external layers as required.

The third way to interact with the system are "data pages", which can be relatively simple views but also full blown applications (Thematic Apps), customized to the user's view on the data provided. Data pages can include visualization and interaction with time series data, photo documentation, document views, spatial previews and many more types of information; data pages can be configured to allow for editing of the data contained as required. 


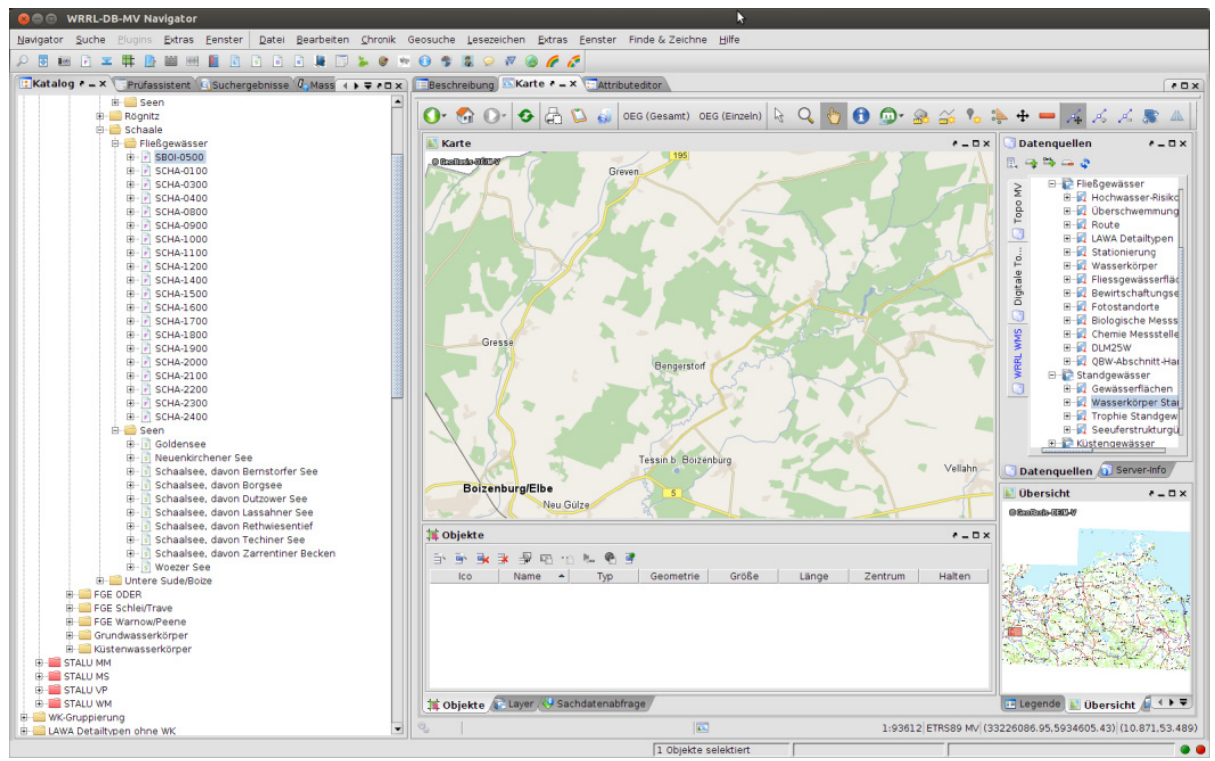

Fig. 6. CIDS Navigator

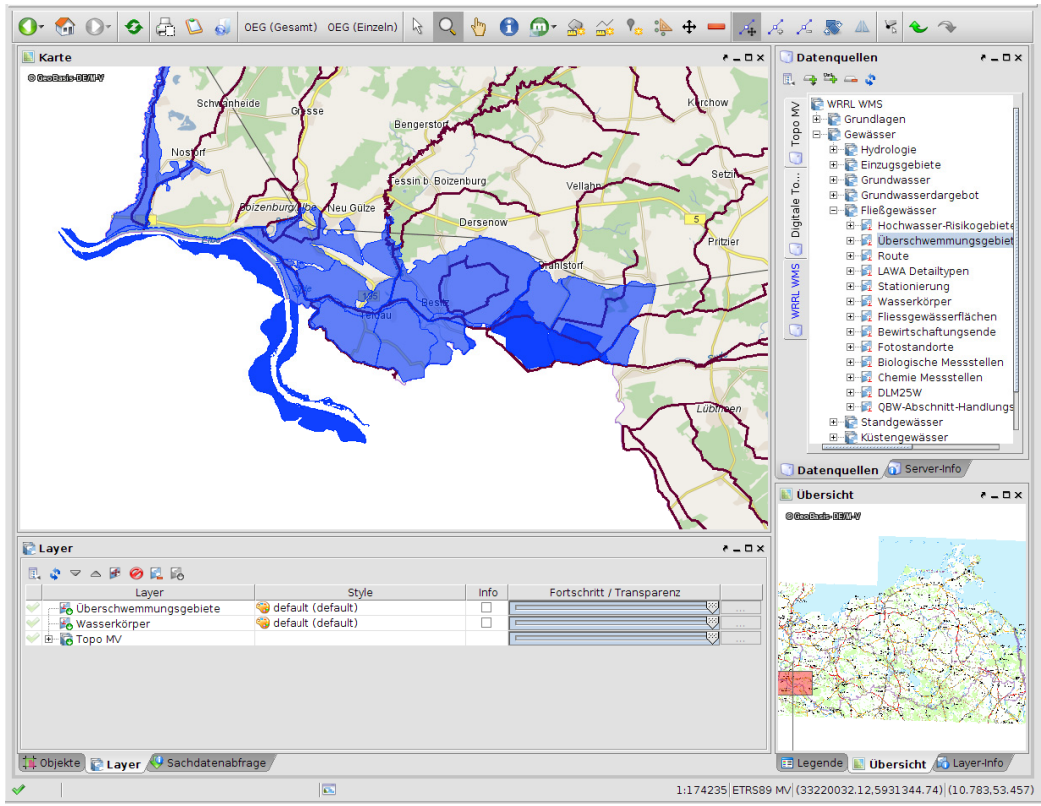

Fig. 7. Geographic interaction with Cismap

An outstanding feature of the Navigator is the fact that users can search, browse and interact seamlessly across these different metaphors. For instance data objects can be dragged on to the map, and polygons on the map can be used to query data themes 
in the service network, including all connected external systems. The software of the Navigator includes extension points for interactive editors and renderers which can be included in map views and data pages respectively. There is also an extension point for information aggregation over a set of objects.

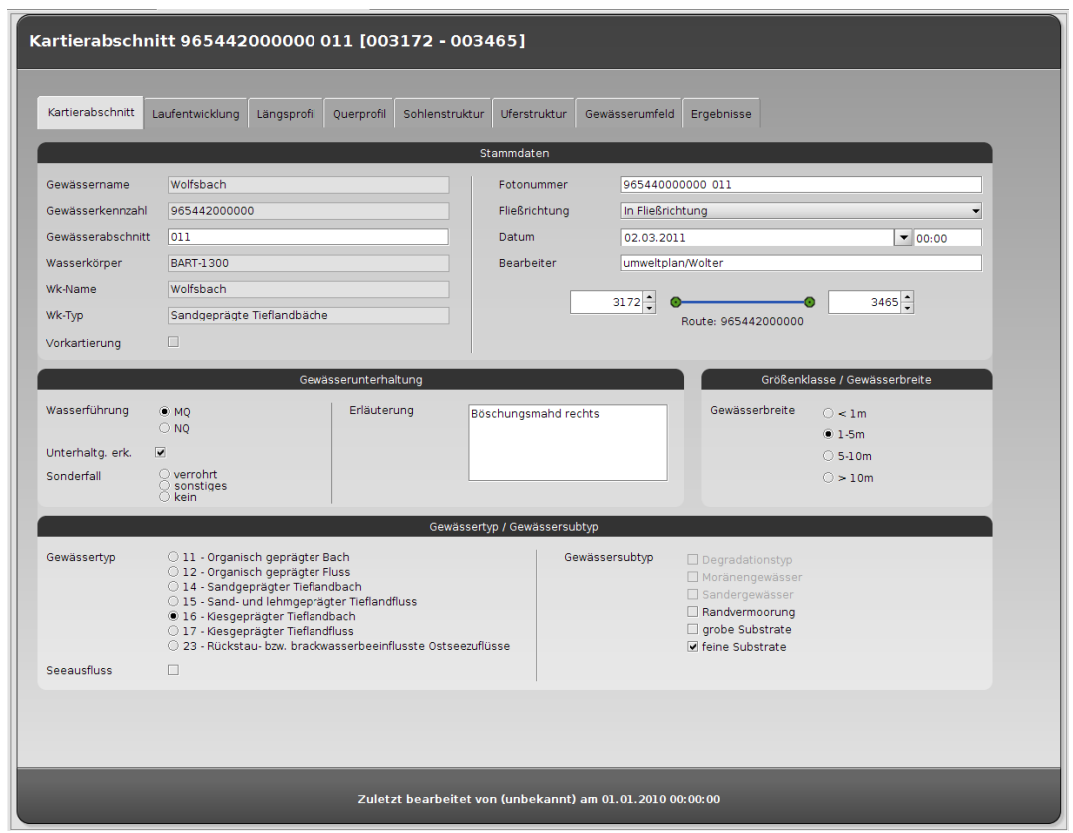

Fig. 8. Thematic view / data editing pages

\section{Customization: Linear Referencing}

The CIDS system provides a powerful framework to build geo-spatial information systems. However, in every project customizations are necessary particularly at the graphical user interface to support end users in an optimal fashion. An example of such a customization is the Linear Referencing implemented as part of the FIS WRRL implementation.

In a river network, the so-called route is an important concept. A route defines one river with a name and a unique number. The river is represented in the system by a polyline " $\mathrm{M}$ " which contains measurement information. Every location along a river can be referenced along the route by the distance to the estuary. This reference is called linear reference. Linear references are used throughout waterway management and are a central concept to connect information to a location (which is along a river). Because this concept is so central, it was essential that end users be supported in stationing (locating) arbitrary objects interactively along a route, and to enable them to create station segments which are for instance used for waterway maintenance projects. 


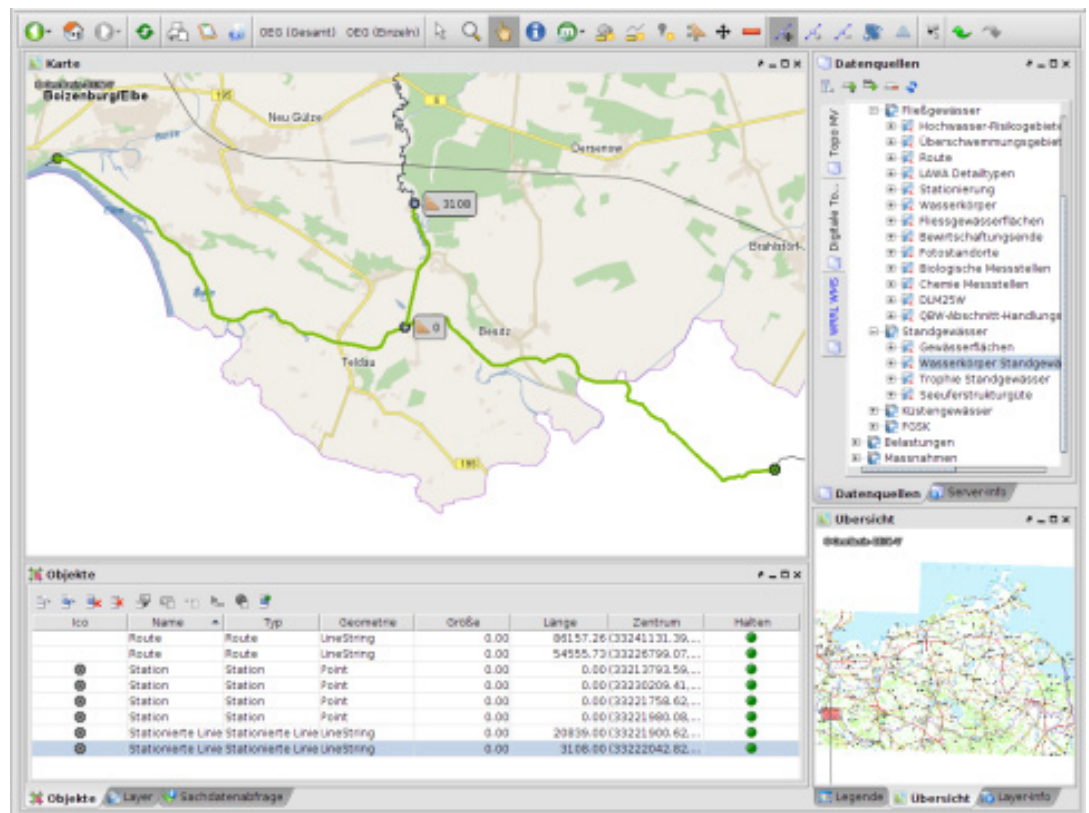

Fig. 9. Linear referencing

Through a custom extension point of CIDS an interactive editor for CISMAP was implemented to support the stationing of objects (Fig. 9). With this, users are enabled to position objects along rivers and to create segments.

\section{$6 \quad$ Assessment and Conclusions}

The FIS WRRL has been in operation since 2011 and has supported WFD reporting since. The FIS Water MV is currently being extended with additional systems in order to provide an integrated water information framework for the state. The choice of open software has proven to be important for long-term protection of investments into the water information infrastructure of the state.

With the realisation of the FIS-WRRL, the state Mecklenburg-Vorpommern has made an important step towards co-ordinated water information management. All WFD-related information can now be managed in a coherent way at a central location while data maintenance is performed in a decentralized fashion by the LUNG, the 4 state agencies and other organisations in a federated fashion based on geographical and organisational access rights.

Now the reports required for EU WFD reporting can be generated in an on-the-fly procedure based on actual daily data.

At the same time, the kernel of an integrated water information system has been developed which serves administration, policy and experts in their daily work. 
The system has been in productive operation in the state agencies for two years, has operated without problems and has become an important information asset for the daily work of experts. Since one year, water management agencies and soil management agencies use the system. In total, 300 users are registered and daily logins are around 70 (May 2013). The users can access a total of 204 information themes supplied by the water management agencies. These themes are structured according to the needs of the WFD. Users generate approximately 15000 map access interactions each day.

The supported functionalities of the system are also extended. Since 2011 a business process supporting the mapping of morphological features of the waterbodies (FGSK - Fließgewässerstrukturgütekartierung) is integrated. Since then, contractors of the 4 state agencies, typically engineering companies, can directly maintain results of the FGSK mapping from their sites. Both contractors and water management agencies thus operate on a highly actual information base.

Currently (June 2013) a solution for river maintenance planning is in the test installation while a project has been started to build an information management application for the inventories of watercourses, profiles and technical plants maintained by the water and soil associations.

The technical solution is fully compliant with OGC standards ${ }^{7}$ and additional information sources complying with these standards can be accessed on-the-fly by end users. Most of these geographical data sources are maps and aerial photos provided by the "Landesamt für innere Verwaltung (LAIV-MV)". Access to mapping services of the neighbouring states Brandenburg, Niedersachsen und Schleswig-Holstein make sure that the view is not restricted at the state borders.

\section{References}

1. eur-lex. europa.eu/LexUriServ/LexUriServ.do?uri=OJ :L:2000:327: $0001: 0072: \mathrm{EN}: \mathrm{PDF}$

2. Güttler, R., Denzer, R., Houy, P.: An EIS Called WuNDa, Environmental Software Systems. Environmental Information and Decision Support, vol. 3, pp. 114-121. Kluwer Academic Publishers (2000)

3. Denzer, R., Torres-Bejarano, F., Hell, T., Frysinger, S., Schlobinski, S., Güttler, R., Ramírez, H.: An Environmental Decision Support System for Water Issues in the Oil Industry. In: Hřebíček, J., Schimak, G., Denzer, R. (eds.) ISESS 2011. IFIP AICT, vol. 359, pp. 208-216. Springer, Heidelberg (2011)

4. Schlobinski, S., Denzer, R., Frysinger, S., Güttler, R., Hell, T.: Vision and Requirements of Scenario-Driven Environmental Decision Support Systems Supporting Automation for End Users. In: Environmental Software Systems. Frameworks of eEnvironment, vol. 9, pp. 51-63. Springer Advances in Information and Communication Technology 359 (2011)

5. Water Information System for Europe (WISE), http: / / water . europa.eu/

7 http://www.opengeospatial.org 